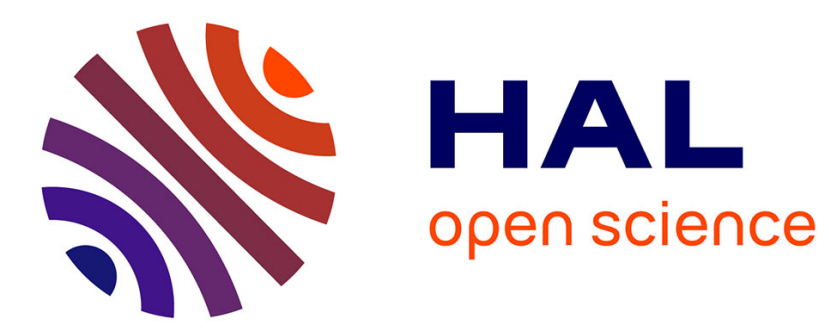

\title{
Combining Structure from Motion and close-range stereo photogrammetry to obtain scaled gravel bar DEMs
}

Wei Li, Stéphane Bertin, Heide Friedrich

\section{- To cite this version:}

Wei Li, Stéphane Bertin, Heide Friedrich. Combining Structure from Motion and close-range stereo photogrammetry to obtain scaled gravel bar DEMs. International Journal of Remote Sensing, 2018, 39 (23), pp.9269 - 9293. 10.1080/01431161.2018.1530809 . hal-03470798

\section{HAL Id: hal-03470798 \\ https://hal.science/hal-03470798}

Submitted on 21 Dec 2021

HAL is a multi-disciplinary open access archive for the deposit and dissemination of scientific research documents, whether they are published or not. The documents may come from teaching and research institutions in France or abroad, or from public or private research centers.
L'archive ouverte pluridisciplinaire HAL, est destinée au dépôt et à la diffusion de documents scientifiques de niveau recherche, publiés ou non, émanant des établissements d'enseignement et de recherche français ou étrangers, des laboratoires publics ou privés. 
Combining Structure from Motion and close-range stereo photogrammetry to obtain scaled gravel bar DEMs

Wei Li (wli860@aucklanduni.ac.nz),

Stephane Bertin (s.bertin@ymail.com), and

Heide Friedrich (h.friedrich@auckland.ac.nz)*

Department of Civil and Environmental Engineering, University of Auckland, Auckland, New Zealand.

* corresponding author (h.friedrich@auckland.ac.nz) 


\section{Combining Structure from Motion and close-range stereo photogrammetry for appropriately scaled gravel bar DEMs}

Digital elevation models (DEMs) have been increasingly applied in topographic studies in areas such as physical geography and hydraulic engineering. Several methods have been proposed to reconstruct DEMs, including classic close-range stereo photogrammetry and the more novel Structure from Motion (SfM) methodology. Past published studies tend to apply SfM to large scale environmental processes, whilst classic close-range stereo photogrammetry is focusing on detailed small-scale applications. However, SfM requires multiple ground control points (GCPs) to allow for proper DEM scaling. The larger the study area, the more GCPs are required, resulting in increased operational complexity and time consuming application of SfM. As the accuracy of the DEM depends on the equipment used to measure GCPs, this can also result in a cost-expensive operation. In the present study, we introduce a combined SfM and close-range stereo photogrammetry application, with the close-range stereo photogrammetry results serving as control for providing scale, thus eliminating the need for traditional GCPs. To validate our methodology, we studied a 40 metre long gravel bar. We used GoPro Hero 3 cameras for SfM measurements and replaced GCPs by DEMs obtained through close-range stereo photogrammetry with a Nikon D5100 camera pair in stereo. In addition to using photo-mode frames, we also studied the quality of DEMs obtained with GoPro Hero 3 video-mode frames, and show how the DEM quality is reduced due to the smaller image format, hence coarser point cloud spacing, which eventually results in a convex curvature when image overlap was increased. Our results show that it is possible to collect high-quality topographic surface data by only using cameras, and alleviate the need for GCPs. The proposed workflow reduces the complexity, time and resource demands associated with deploying GCPs and necessary independent geo-referencing, ensuring that digital photogrammetry will continue to gain popularity for field surveying.

Keywords: remote sensing, photogrammetry, SfM, river hydraulics, gravel beds, roughness 


\section{Introduction}

With new papers published regularly, the popular use of light-weight and inexpensive yet effective remote-sensing techniques in Earth Science is evident (e.g., Carrivick et al. 2013; Fonstad et al. 2013; James and Robson 2012; Westoby et al. 2012). Common to a number of applications is the necessity to measure landforms, and their variability through time and space, as this is critical for understanding surface processes. In river hydraulics, using digital elevation models (DEMs) has become more attractive (i) to reveal the flow history that shaped the surface (Ockelford and Haynes 2013), (ii) to parameterise surface roughness for flow resistance modelling (Smart, Duncan, and Walsh 2002) and (iii) to quantify sedimentation patterns (Lane, Westaway, and Hicks 2003). Additionally, gravel bars are important for the rivers' ecology (Hauer et al. 2014) and play an important role in channel evolution (Rice and Church 2010). Gravel bars are difficult to study, in part because they are made up of difficult terrains in a dynamic environment, with a large size $\left(10-1000 \mathrm{~m}^{2}\right)$ and surface heterogeneity (Buffington and Montgomery 1999). Traditionally, obtaining quantitative data was often limited to manual sediment size measurements (Church, McLean, and Wolcott 1987; Hassan 2005).

Presently, continuous progress in remote sensing is transforming field surveying. Several traditional limitations related to accessibility, terrain disruption, time and costs can be overcome nowadays, whilst at the same time improving both the spatial coverage and spatial and temporal resolution of measurements (e.g., Entwistle and Fuller 2009; Heritage and Milan 2009). Image-based methods (e.g., digital photogrammetry) present advantages, such as quick data acquisition over a diverse range of distances. This results in an accuracy that is similar to terrestrial laser scanners (TLS) and other LiDARs, and the possibility to operate at higher resolution (Bertin and Friedrich 2014; Morgan, Brogan, and Nelson 2017; Westoby et al. 2015). There is also the potential for light, low 
bulk and budget equipment to form the photogrammetric toolkit, making the technique suitable for use in difficult terrains (Bird, Hogan, and Schwab 2010; Rieke-Zapp, Rosenbauer, and Schlunegger 2009), as well as on board light-weight UAVs (De Souza et al. 2017; Marteau et al. 2017; Westoby et al. 2015).

Using digital photogrammetry in the field is becoming more prevalent, for various applications, including fluvial landscape measurement at both grain- (Bertin and Friedrich 2016; Wang, Chung, and Lin 2015) and river-reach scale (Javernick, Brasington, and Caruso 2014; Woodget et al. 2015), characterisation of agricultural lands (De Souza et al. 2017; Ouédraogo et al. 2014) and Antarctic moraines (Westoby et al. 2015), as well as time-lapse monitoring of active lava flows (James and Robson 2014), braided river channels (Lane, Westaway, and Hicks 2003) and beach morphometry (Brunier et al. 2016). Recent applications essentially use consumer grade digital cameras for image acquisition, whereas image processing is done using a variety of methods. In general, two broad approaches can be distinguished for photogrammetric image processing: (1) stereo photogrammetry, whereby stereo-matching of corresponding pixels between two images with significant overlap allows reconstruction of a depth (also called disparity) map, which can be transformed into a metric point cloud and subsequent DEM, by using scaling information provided through calibration; and (2) Structure from Motion (SfM) photogrammetry, which makes use of a large number of images to reconstruct camera motion and scene rendering without the need for calibration, whilst scaling is commonly provided by ground control points (GCPs) and independent measurements.

Below, we present the two approaches in more detail to highlight current limitations and to motivate our research efforts of combining SfM and close-range stereo photogrammetry to perform measurements of a gravel bar using just cameras. 


\subsection{Stereo photogrammetry}

In order to accurately reconstruct distances and topography with stereo photogrammetry, camera parameters, both internal (e.g., focal length and distortion coefficients) and external (e.g., translation and rotation between exposure locations), are measured through calibration. 'Self-calibration' techniques have the advantage that they only require the acquisition of imagery used for the DEM extraction. Numerous (minimum recommended of 15) GCPs are evenly distributed throughout the $x, y$ and $z$ volume of the study site and are recorded with an independent device, generally a GPS (Rumsby et al. 2008) or a total station (Bird, Hogan, and Schwab 2010; Chandler, Fryer, and Jack 2005). Limitations relate to the duplicity of equipment brought on site, eventual terrain disruption when disposing and geo-referencing GCPs, and the need to repeat the procedure for each measurement location. Besides, GCP localisation errors, which can be large and depend on the instrumentation used, propagate into the photogrammetric workflow and affect final DEM accuracy (Carbonneau, Lane, and Bergeron 2003; Chandler et al. 2001). In comparison, 'test-field' calibration techniques require additional images of a specific target of known dimension to be recorded. In this case, the stereo system, generally comprising two cameras rigidly connected, is designed prior to calibration and both the baseline distance between cameras and camera aperture are kept constant throughout the application. Bertin et al. (2015) presented a complete workflow, whereby the acquisition of 30 images of a chequerboard moved across the 2D field of view (FoV) between overlapping images, thus minimising the risk of systematic calibration errors. Bertin and Friedrich (2016) used this method in the laboratory prior to measuring gravel bars in the field. Following careful transport of the camera setup, calibration data were used successfully for sub-millimetre accurate DEM collection at multiple locations. The application required pre-supposition of the camera-to-object distance (mainly because of fixed camera focus and aperture after calibration), which restrained measurements to 
small $\left(<1 \mathrm{~m}^{2}\right)$ gravel patches. According to the authors, measuring a complete gravel bar in this way would require merging numerous DEMs, individually collected and overlapping, which is both difficult to implement and requires long data collection and processing time.

Furthermore, the accuracy of the final DEM relies on appropriate stereo-matching of corresponding pixels, whilst image resolution and image quality ultimately control DEM quality (Bertin et al. 2015). For this reason, stereo photogrammetry prefers highquality DSLRs with a large image format, whilst well-balanced and contrasted images prevent random matching errors (Bertin et al. 2015; Mosbrucker et al. 2017). However, variable lighting conditions observed in the field generally challenge the recording of 'good-quality' images with fixed aperture, requiring substantial image post-processing (Bertin and Friedrich 2016).

Finally, appropriate camera synchronization is critical. Small areas can be surveyed with static camera stands (e.g., tripods), allowing manual camera synchronization (Bertin and Friedrich 2016). However, as the camera height increases, lighter camera stands are required. For applications, such as comprehensive gravel bar coverage, keeping the viewing geometry still, whilst acquiring stereo images, is problematic. Thus, although accurate calibration data might be available, due to unstable camera frames and inaccurate synchronisation, an accurate DEM reconstruction might not be achieved.

\subsection{Structure from Motion (SfM) photogrammetry}

Novel Structure from Motion (SfM) and Multi-View Stereo (MVS) methods allow using one camera and a large number of images, collected from various viewpoints and distances, for DEM reconstruction. This alleviates the needs for camera calibration and 
restrictive pre-defined setup geometries. It also provides the opportunity for using lower cost and lower resolution cameras.

SfM performs sparse point cloud reconstruction by matching features across images. The majority of applications then apply MVS algorithms to increase point density (hence the appellation SfM-MVS). However, MVS contribution is mainly to expand feature matching according to the sparse features initially detected, and thus is not able to create a similar dense point cloud as if stereo-matching corresponding pixels is used. Instead, it is common to increase the image quantity to allow for more features to be matched. For instance, James and Robson (2012)'s high-resolution (point spacing < 1 $\mathrm{mm}$ ) measurement of a volcanic rock (length $\sim 0.1 \mathrm{~m}$ ) used 92 images for DEM reconstruction, which took $\sim 12$ hours of reconstruction. Despite seemingly complex multiple image acquisition and processing, the automation and convenience afforded by software such as Agisoft PhotoScan and VisualSfM generally balance disadvantages centred on time and resource demands for multi-view feature matching. Besides, previous work has shown that feature matching can perform well even in sub-optimal lighting conditions (Mosbrucker et al. 2017), which provides advantages of using SfM in the field.

Using un-calibrated imagery however raises the issue of measurement scaling needed for quantitative analysis. Scale is generally provided using pre-surveyed (i.e. independently measured) GCPs (e.g., Woodget et al. 2015), however the same GCPs issues apply as discussed for stereo photogrammetry (see previous section). In practice, at least six uniformly-distributed GCPs per image overlap are recommended, although three GCPs are suitable in theory. Besides GCPs quantity, the size, shape and colour of GCPs, as well as geo-referencing accuracy, are all critical factors affecting DEM quality (Stamatopoulos and Fraser 2014). For instance, at-a-point GPS positioning can achieve 
$10 \mathrm{~mm}$ accuracy; however, the error count after bundle-adjustment using multiple GCPs can ultimately reach ten-fold.

Albeit limited, examples of scaling without GCPs are found in the literature. Morgan, Brogan, and Nelson (2017) used CloudCompare (Girardeau-Montaut 2011) and ICP algorithms in combination to align and scale SfM with existing TLS recordings in laboratory flumes. Perhaps more crude and limited to small scales, yet able to achieve excellent results, James and Robson (2012) used a steel ruler (RMS error of $110 \mu \mathrm{m}, \mathrm{n}=$ 6) and easily-identified features. Finally, recent work by Carbonneau and Dietrich (2017) illustrates how continuous technical improvements, such as the advent of cameras embarked with dGPS, may well ultimately eliminate the need for GCPs in future applications.

\subsection{Paper overview}

This paper builds on previous efforts to further the use of digital photogrammetry in the field. We present two applications in tandem: (i) measuring an exposed gravel bar $(\sim 200$ $\mathrm{m}^{2}$ ) with SfM, using a GoPro Hero 3 camera and (ii) stereo photogrammetry using two Nikon D5100 cameras to obtain detailed DEMs of sections of the bar $\left(\sim 1 \mathrm{~m}^{2}\right)$, called patches. A combined SfM and close-range stereo photogrammetric workflow is proposed to use the DEMs obtained with stereo photogrammetry as control to properly scale SfM results. The final products are accurate DEMs at both bar- and patch scale, for which georeferencing to a global coordinate system, although not necessary in our study, can be implemented in a second step. The originality of our workflow is due to the fact that only digital cameras are required to obtain accurate 3D measurements in the field, no GCPs are needed. Thus, by not using GCPs, we show that a high-quality DEM accuracy can be obtained by reducing recording as well as processing time, as well as introducing a new cost-effective field measurement technique. Our quantitative DEM evaluation shows that 
the proposed workflow represents a viable alternative to current SfM approaches used in Earth Sciences. We provide guidelines and considerations for future use and discuss our study in relation to other applications.

\section{Methods}

\subsection{Field site and data collection}

The Whakatiwai River, a small gravel-bed stream in New Zealand's North Island, was the location for our field tests. Presented measurements focus on one exposed gravel bar, located 300 metres upstream from the stream mouth. This gravel bar was chosen due to easy equipment access from the road, and because previous field investigations were undertaken on this gravel bar (Bertin and Friedrich 2016; Groom, Bertin, and Friedrich 2018; Stähly, Friedrich, and Detert 2017). The gravel bar was attached to a densely vegetated bank. The surface of the bar was vegetated upstream with pampas grass, and there was a change in elevation towards the water edge, with a slope vegetated by grass. Complete data collection was performed during one sunny day, which was timed to coincide with low flow condition, with most of the gravel bar exposed in this case.

For collecting data, a $30 \mathrm{~m}$ tape measure was placed alongside the bar, and care was taken to only walk along this transect in order to not destroy natural sedimentation patterns. 14 gravel patches (Figure 1) were systematically chosen, either side of the transect on non-disturbed areas, to cover a down-bar and lateral pattern. At each patch, stereo images were collected using two Nikon D5100 cameras (16.4 Mpixel, $23.6 \times 15.6$ $\mathrm{mm}^{2}$ sensor size) with Nikkor $20 \mathrm{~mm}$ fixed-focus lenses in stereo (baseline distance of $0.3 \mathrm{~m})$, from a vertical distance of approximately $0.75 \mathrm{~m}(\mu=0.744 \mathrm{~m}, \sigma=0.008 \mathrm{~m}, \mathrm{n}=$ 44, where $\mu$ and $\sigma$ are mean camera distance and standard deviation determined after DEM reconstruction, respectively, and $\mathrm{n}$ is the total number of stereo pairs collected). 
The same setup was used previously by Bertin and Friedrich (2016) for measuring gravel patches in the field. Cameras were rigidly connected by a metallic rail installed horizontally above the exposed riverbed using two tripods. The rail was long enough to prevent tripods from casting shadows or appearing in the image overlap where 3D is reconstructed. Cameras were connected to a laptop allowing remote control, such as adjusting the shutter speed and synchronising image acquisition. Fixed focus was established beforehand in the laboratory at a distance of $\sim 0.7 \mathrm{~m}$, and aperture was maintained constant to $\mathrm{f} / 20$ throughout the application. For the majority of patches, multiple (up to 5) stereo pairs were collected with sufficient overlap (aim was $\sim 30 \%$, established in the field using a steel ruler) to allow merging individual DEMs, therefore extending the patch surface measured. With a camera distance of $0.75 \mathrm{~m}$, minimum surface coverage was $0.60 \times 0.60 \mathrm{~m}^{2}$ when using one stereo pair (see Figure 2 for representation), which extended to a maximum measured size of $1.85 \times 0.38 \mathrm{~m}^{2}$ after merging individual DEMs. The overlap between the two images forming a stereo pair was $\sim 67 \%$ (not to confound with the $30 \%$ overlap between successive DEMs allowing merging), whilst horizontal (i.e. pixel size) and vertical (i.e. depth) measurement resolution was $0.19 \mathrm{~mm}$ and $0.47 \mathrm{~mm}$, respectively. Exact values varied slightly in relation to the small variations in camera-to-riverbed distances presented above. Previous work using the same setup in the field found DEM accuracy to be $\sim 0.7 \mathrm{~mm}$ (Bertin and Friedrich 2016). DEM accuracy was computed as the mean unsigned error (MUE) between measured and true elevations using a 3D-printed ground truth resembling a water-worked gravel bed. 


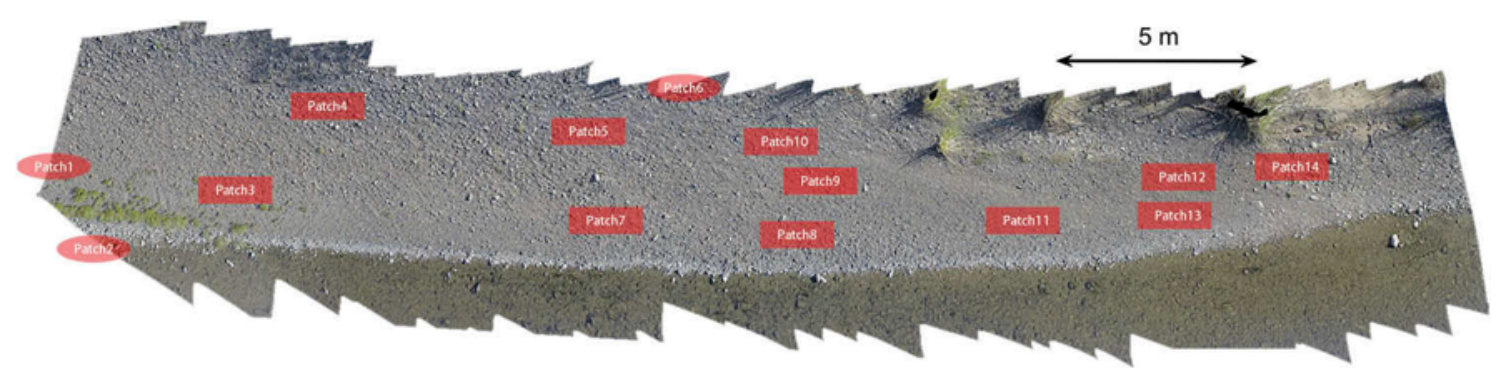

Figure 1. Ortho-image of the bar obtained with GoPro Hero 3 (video mode) and the SfM workflow, showing the location of the 14 gravel patches measured independently, using stereophotogrammetry. Flow direction is from left to right. Circled patches fall outside SfM measurements performed in photo mode.

Obtaining photographs for the 14 patches took approximately one hour, after which a single GoPro Hero 3 mounted on a pole was used to capture images of the bar from a vertical distance of approximately $2 \mathrm{~m}$ (Figure 2). The small size and large field of view (horizontal FoV $=61$ degrees) of the GoPro Hero 3 allowed convenient operation and complete bar-width coverage from a single exposure location. Covering the length of the bar was achieved by walking slowly and steadily in the down-bar direction, while maintaining camera orientation to near vertical throughout. The bar was traversed alongside twice, allowing using both photo and video modes for recording. Data acquisition was controlled using the GoPro application for iPad. Photo mode provides a greater image quality $(4000 \times 3000$ pixels $)$, while video is recorded at $48 \mathrm{fps}$ and $1920 \times$ 1440 pixels. The two modes are characterised by the same FoV. Previous SfM applications with GoPro used photo mode exclusively (e.g., Marteau et al. 2017), due to the larger image format. The possibility to collect data 'on the go' with maximum fluidity motivated testing the video mode in this study. Complete data collection took less than 15 minutes, including collecting a one-minute video. Examination of the data was carried out in the office to remove redundant images and to extract video frames. A total of 32 
photos and 317 video frames (out of originally 3263 frames, which corresponds to keeping approximately one in every ten frames) were retained for SfM processing.

\subsection{SfM workflow for GoPro Hero 3 imagery}

Processing the GoPro Hero 3 images was performed with the commercial software Agisoft PhotoScan V.1.2.7. The latter contains a complete integrated workflow, from camera calibration to DEM reconstruction, through computations of sparse point cloud, dense point cloud, mesh and texture.

Camera calibration is critical for fish-eyed lens cameras, such as GoPro Hero 3 (James and Robson 2012; Marteau et al. 2017). 29 images of a chequerboard ( $1 \mathrm{~m}^{2}, \sim 2$ $\mathrm{Kg}$, square size $=50 \mathrm{~mm}$ ) were collected whilst in the field and were later processed with Bouguet (2010)'s open-access calibration toolbox for Matlab, allowing both photos and video-frames to be un-distorted.

After un-distortion, photos and video-frames were processed separately in Agisoft, following the same steps: (i) sparse point cloud computation, whereby feature extraction and matching across images is performed with SIFT; (ii) dense point cloud computation using Agisoft's MVS algorithm; (iii) mesh computation; (iv) texture computation providing the ortho-image (e.g., Figure 1); and finally (v) scaled point cloud reconstruction and interpolation as a raster DEM. For the latter step, the DEM of a gravel patch measured independently with stereo photogrammetry was used as reference for providing a scaled coordinate system (see next section).

Parameter setting is an important aspect when using Agisoft. We set the sparse point cloud quality to "highest" (out of the options "lowest", "low", "medium", "high", and "highest"), which allowed using the best image resolution possible during feature matching. Subsequently, we set the dense point cloud quality to "ultra-high" (out of the options "lowest", "low", "medium", "high", and "ultra-high") to achieve maximum point 
density (Morgan, Brogan, and Nelson 2017). Resulting large computational efforts were afforded by high-performance computing available at the University of Auckland, with access to a virtual machine with 12 cores CPU and 32 GB memory.

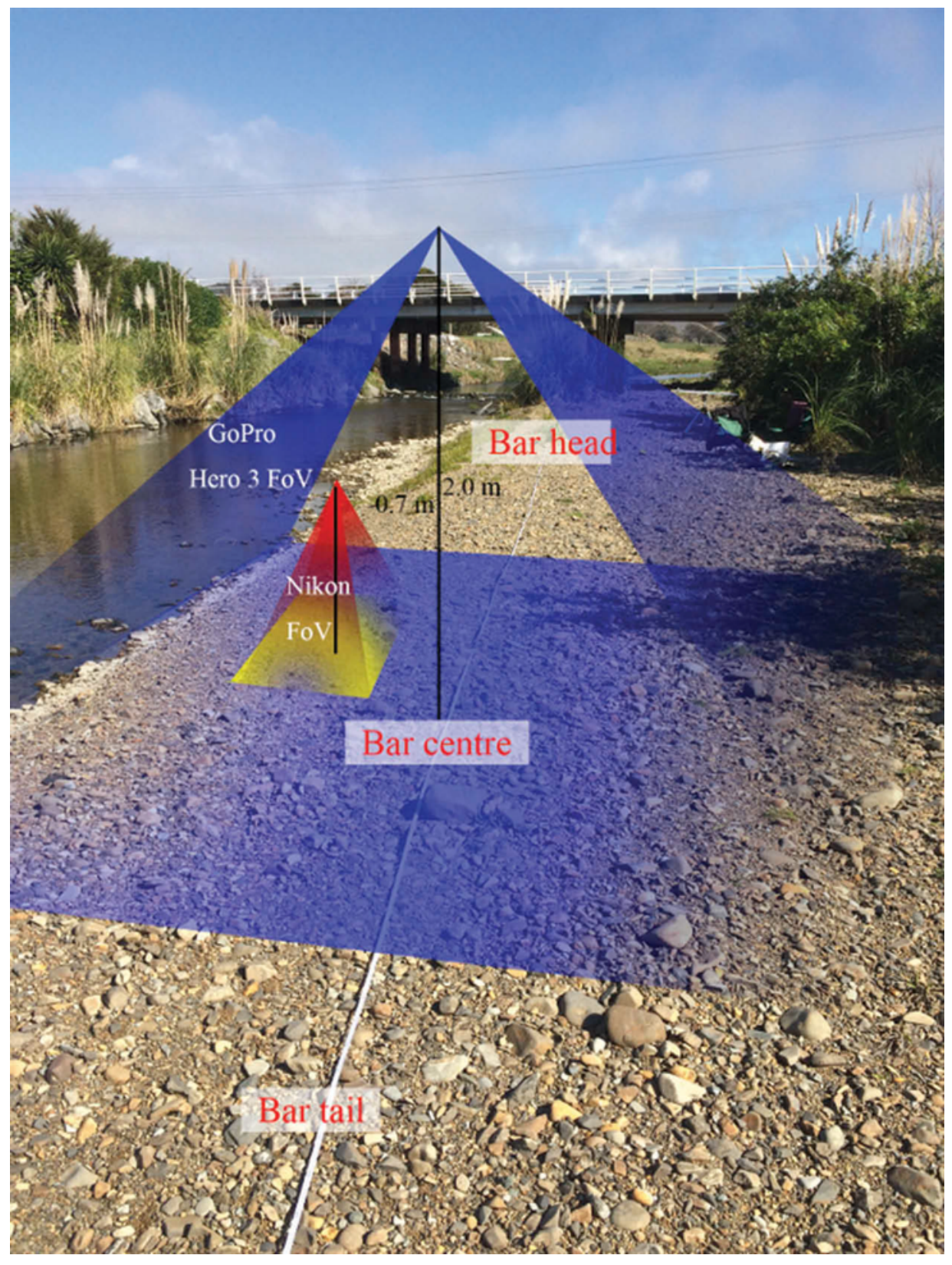

Figure 2. Schematic presentation of GoPro Hero 3 and Nikon D5100 field image acquisition, showing different fields of views (FoVs) resulting from varying camera distance and equipment. 


\subsection{Stereo photogrammetry workflow for Nikon D5100 imagery}

Stereo images collected with the Nikon D5100 cameras were processed using a nonproprietary stereo photogrammetry workflow developed by the authors, as it allows highresolution and high-accuracy measurements over gravel patches (Bertin and Friedrich 2016; Groom, Bertin, and Friedrich 2018). This provided reliable ground truth data, both for dimension-scaling and evaluating SfM measurements.

The photogrammetric technique employed herein to obtain DEMs from stereo photographs (i.e. two overlapping images) consists of (i) in-situ calibration, using the method of Zhang (1998), included in Bouguet (2010)'s open-access calibration toolbox for Matlab, which requires several stereo photographs of a planar chequerboard to determine both internal and external calibration parameters; (ii) using the calibration data to rectify stereo photographs of the gravel to epipolar geometry, whereby corresponding pixels between overlapping images are ideally on a same scanline (i.e. same vertical pixel coordinate); (iii) scanline-based pixel-to-pixel dense stereo matching using Gimel'farb (2002)'s symmetric dynamic programming stereo (SDPS) algorithm, providing both scaled point clouds and ortho-images; and (iv) interpolation (using the triangle interpolation method in Matlab) as raster DEMs with final grid spacing equal to $1 \mathrm{~mm}$.

In practice, we collected calibration images both in the laboratory, the day before, and in the field. Each time, 30 stereo photographs were recorded, with the chequerboard thoroughly moved across the $2 \mathrm{D}$ FoV between overlapping images. Repeating the calibration was motivated by our previous field applications (Bertin and Friedrich 2016) that showed that careful transport of the stereo setup is critical to calibration stability and thus DEM quality. We evaluated the rectification error (i.e. deviation from perfect epipolar geometry) for both laboratory and field calibrations using five independent calibration images collected in the field. Results showed sub-pixel accurate calibrations 
( $\mu=0.47$ and $\sigma=0.18$ compared to $\mu=0.88$ and $\sigma=0.40$, for field and laboratory, respectively). Consequently, the decision was made to use the field calibration for analysis, motivated by the smaller rectification error.

DEM merging increased surface coverage at most patch locations. We used our own seamless DEM merging algorithm coded in Matlab, whereby $(\sim 30 \%)$ overlapping DEMs were aligned through horizontal and vertical translations, and 3D solid rotations, before merging (Bertin, Friedrich, and Delmas 2016). Overlapping elevations were averaged giving equal weight to both DEMs, and points considered as outliers (here points outside the elevation range of $\mu \pm 3 \sigma$ ) were replaced using bi-cubic spline interpolation. Concurrently, ortho-images were processed to allow draping texture over the DEMs. Textured DEMs allowed easy and precise feature identification, which was found important for using stereo photogrammetry for providing scaled coordinates for SfM.

\subsection{SfM dimension scaling using stereo photogrammetry}

Dimension scaling and establishing a coordinate system in SfM represents the last step of the computation. Traditionally, (physical) GCPs distributed across the study site are measured independently, and resulting coordinates are attributed to GCP locations identified in Agisoft (e.g., Brunier et al. 2016; Woodget et al. 2015). In this study, a DEM of a gravel patch measured with stereo photogrammetry is used as control for providing accurate scaled coordinates for SfM. For that, a point-point correspondence between the two datasets is established by manually selecting riverbed features (hereafter referred to as "control points (CPs)) easily identifiable with both stereo photogrammetry and SfM (Figure 3). In Agisoft, the process of using CPs is equivalent to the classical use of GCPs, making for an integrated workflow. Markers are created for each CP, and optimisation is carried out by manually selecting the corresponding locations in all relevant images. We 
note that because DEMs obtained with stereo photogrammetry were not referenced to a common coordinate system (as this would require (physical) GCPs and independent georeferencing), establishing a point-point correspondence between stereo-photogrammetry and SfM cannot be done simultaneously across multiple patches. Instead one reference patch (e.g. patch 9) is used to provide a number of CPs.
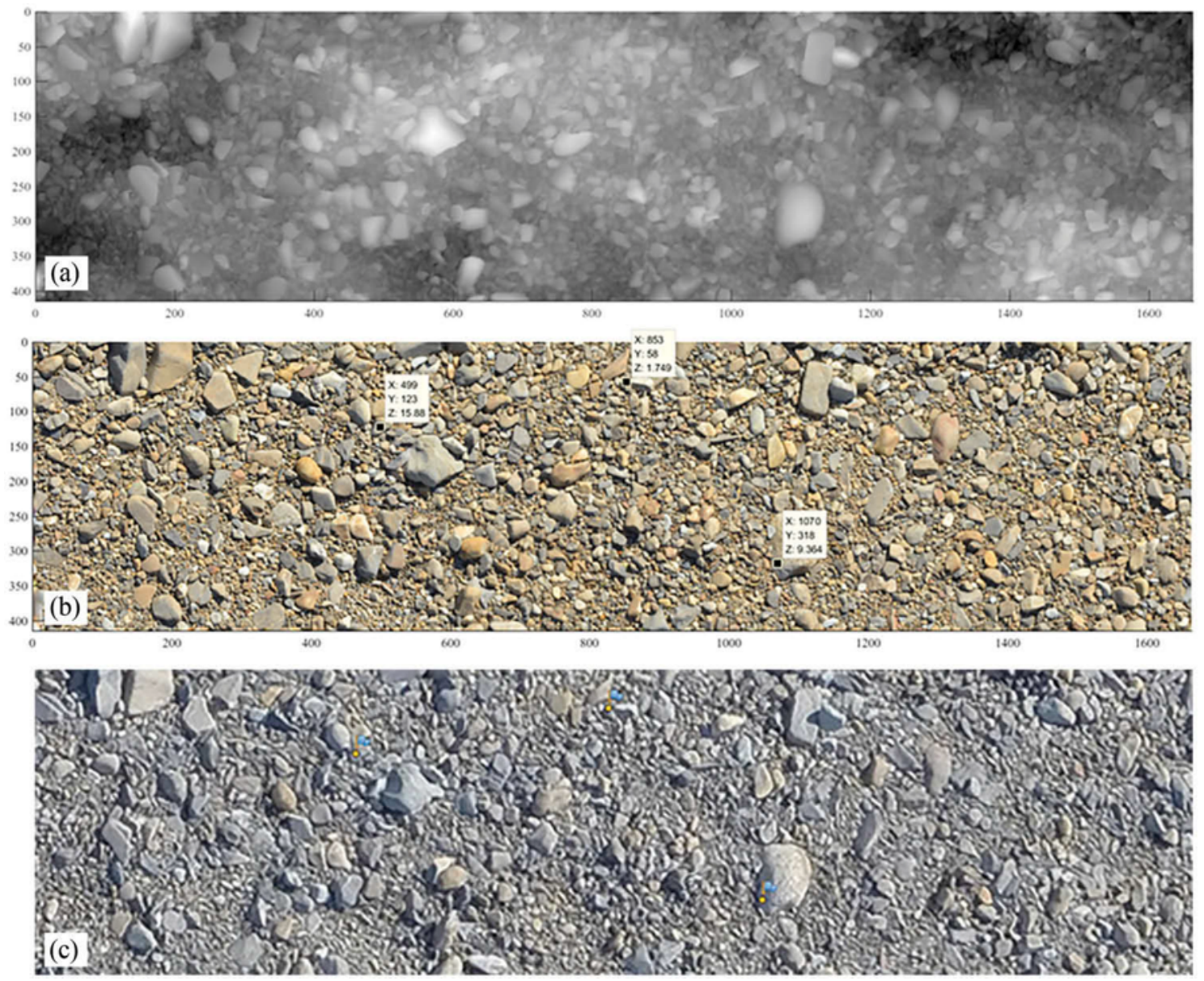

Figure 3. The process of establishing CPs for SfM dimension scaling. (a) un-textured stereo photogrammetry DEM; (b) selected CPs and the textured stereo photogrammetry DEM; and (c) markers created at the location of the CPs in Agisoft. Dimensions are in millimetres.

Table 1. Characteristics of SfM datasets, including image quantity, image format, surface coverage, and sampling resolution (i.e. point spacing).

\begin{tabular}{lcccc}
\hline & Image count $(-)$ & Image format (pixel) & Sampling resolution $(\mathrm{mm})$ & Coverage $\left(\mathrm{m}^{2}\right)$ \\
\hline Photo mode & 32 & $4000 \times 3000$ & 3.3 & 240 \\
Video mode & 317 & $1920 \times 1440$ & 2.9 & 214 \\
\hline
\end{tabular}




\subsection{SfM error evaluation and summary of the tests}

For testing DEM quality (e.g., the accuracy) achieved with SfM, we used the DEMs obtained with stereo photogrammetry as benchmarks. The method is as follows. A DEM of the complete gravel bar obtained with SfM is cut into smaller DEMs encompassing the patches measured with stereo photogrammetry. A Matlab program was developed for aligning the SfM DEMs with the 'truth DEMs' obtained with stereo photogrammetry. For each patch, the initiation is the selection of two corresponding points in the DEMs, allowing rapid horizontal alignment by translation and 2D rotation. Optimisation is then performed automatically by means of translation (using grid spacing as the increment) and $3 \mathrm{D}$ rotation until the difference between DEMs is minimised. We used the mean unsigned error (MUE) between DEMs as the discriminant for best alignment. After alignment, SfM and stereo photogrammetry are compared point-by-point over a $1 \mathrm{~mm}$ grid, providing error statistics and a DEM of difference (DoD). To ensure minimum effect from DEM (mis-)alignment on the error statistics presented, all comparisons were repeated twice and error statistics were retained at the condition of consistent results. The procedure was repeated for all patches measured with stereo photogrammetry, allowing identification of SfM-error spatial distribution across the gravel bar. Using a $1 \mathrm{~mm}$ grid meant SfM measurements were interpolated onto grids with spacing smaller than initial point spacing in point clouds (see Table 1). Thus, part of the errors determined from comparing SfM DEMs with benchmarks may be attributed to interpolation. However, there was a critical advantage to using $1 \mathrm{~mm}$ grid spacing, in that it benefits the reliable comparison of SfM DEMs with benchmarks, compared to using larger grid spacing, as alignment precision is commensurate with grid spacing (Bertin, Friedrich, and Delmas 2016). We note that 'over-interpolation' was used before by Hodge, Brasington, and Richards (2009) for analysing laser-scanned DEMs of gravel-bed surfaces. In their study, 
elevations were interpolated onto $1 \mathrm{~mm}$ grids despite the laser footprint being $4 \mathrm{~mm}$ and no effect on the analysis was observed.

Using the evaluation method presented above, we tested the effect of CP quantity and the effect of varying the reference patch on resulting SfM DEMs. We first used patch 9, located in the bar centre (Figure 1), as the reference stereo photogrammetry DEM, and we compared results obtained using 3 and $10 \mathrm{CPs}$. The number of CPs tested was selected both to equal and surpass the minimum recommended, whilst still ensuring timely operation. We then used patch 3 (Figure 1), located at the bar edge, as the reference patch for providing CPs. In this case, 3 CPs were used.

SfM reliability was also assessed by comparing complete (i.e. whole-bar) DEMs obtained using (i) different modes of data collection (e.g., photo and video modes); (ii) varying numbers of CPs (e.g., 3 and $10 \mathrm{CPs}$ ); and (iii) different reference patches (e.g., patch 3 and patch 9). The same alignment procedure as presented above was used before point-by-point comparison. However, using $1 \mathrm{~mm}$ grid spacing was not possible due to the large DEM size and resulting unmanageable computing demands. We used a grid with $20 \mathrm{~mm}$ spacing, as it provided a good balance between DEM resolution hence alignment precision, and computing time. DEM grid resolutions of between 20 and $50 \mathrm{~mm}$ were used by others before for studying riverbed morphology (Marteau et al. 2017; Woodget et al. 2015).

\section{Results}

\subsection{Comparison between GoPro Hero 3 photo- and video-mode}

Figure 4 shows scaled ortho-images and DEMs of the gravel bar resulting from processing separately the GoPro Hero 3 photos and video-frames with SfM. Surface coverage is $(\sim 10 \%)$ larger in the case data were recorded in photo mode (Table 1, Figure 
4). The difference in coverage is essentially due to the DEM reconstructed using photos (hereafter called the photo-DEM) being larger in the direction transverse to the bar and covering inundated areas which are not included in the video-DEM. Bar-length coverage is however more important in the video-DEM, with a maximum length of $41.4 \mathrm{~m}$ compared to $36.1 \mathrm{~m}$ for the photo-DEM (Figure 4).

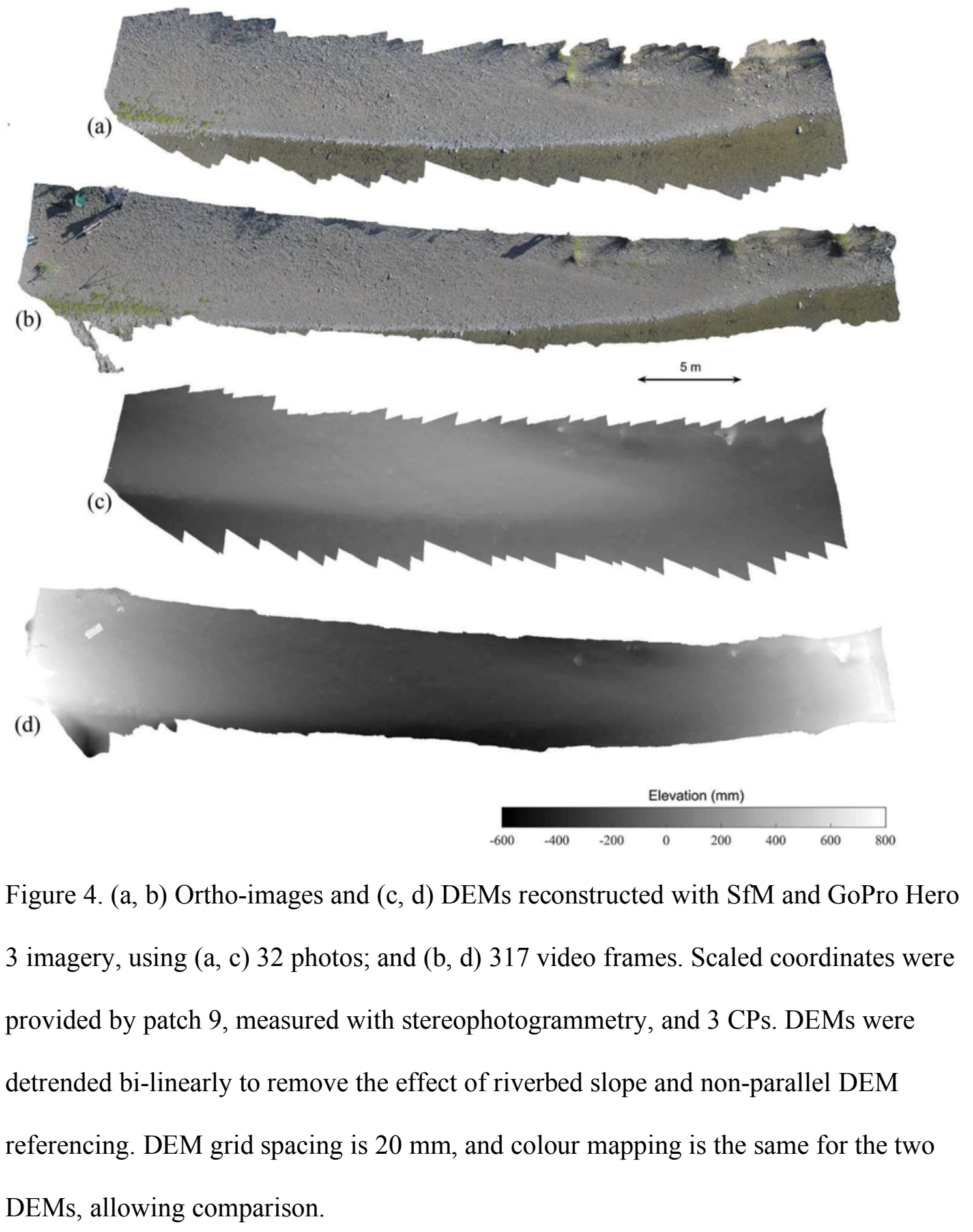


DEMs (Figure 4C and 4D) were previously detrended to remove the combined effects of the general riverbed slope and DEM referencing to a coordinate system based on patch 9 measured with stereo photogrammetry, by fitting a bi-linear surface using the least-squares method (Bertin, Friedrich, and Delmas 2016). This enabled gravel-bar morphology, initially concealed by larger scale elevation trends, to be revealed. Visually, our field observations of an elevated bar platform gradually sloping down towards the bar tail, are evident in the photo-DEM (Figure 4C). Larger surface coverage transverse to the bar for the photo-DEM allows inundated areas to be represented as regions of low elevation. In this work no attempt was made to correct for the refraction of light rays at the air-water interface, which normally prevents true submerged bed elevation determination with optical measurements (Bertin et al. 2013; Woodget et al. 2015). This is because data collected are destined for analysis of gravel-bar roughness and sedimentation patterns, therefore focusing on exposed areas. Besides, water surrounding the bar was shallow $(<0.2 \mathrm{~m})$, and refraction correction procedures were found unnecessary in this case (Woodget et al. 2015).

Visual observation of the video-DEM shows a contrasting bar morphology globally (Figure 4D), whereby the bar platform is concealed by high elevations both upstream and downstream of the bar. These high elevations, together with low elevations in the bar centre, may indicate the presence of convex curvature in the video-DEM.

DEMs obtained with photo- and video-mode, respectively, are compared in Figure 5. For producing the DEM of difference (DoD) presented in Figure 5A, the photoDEM was subtracted (point-by-point on a $20 \mathrm{~mm}$ grid) from the video-DEM after mutual alignment. Convex curvature of the video-DEM is evident in the DoD. If we consider the photo-DEM as reference, any difference between the photo- and the video-DEM can be attributed to errors in the video-DEM. This way we measured a mean unsigned error 
(MUE) of $0.249 \mathrm{~m}$, standard deviation of error $\mathrm{SDE}=0.291 \mathrm{~m}$ and a maximum error in absolute of $1.028 \mathrm{~m}$. For allowing sole characterisation of errors resulting from convex curvature, a bi-quadratic trend was fitted to the DoD by least squares (Figure 5B). Using the trend surface, we found $\mathrm{MUE}=0.244 \mathrm{~m}$ and $\mathrm{SDE}=0.286 \mathrm{~m}$, which indicates that the curvature of the video-DEM is the main reason for the difference between photo- and video-DEMs.

\subsection{Effect of CP quantity}

For this part of the analysis, SfM DEMs obtained using photo mode, patch 9 as the reference patch, and varying numbers of CPs, are cropped to produce 11 DEMs corresponding to the 11 gravel patches measured independently with stereo photogrammetry. We note that 14 gravel patches were measured with stereo photogrammetry, of which only 11 are contained in SfM measurements carried out in photo mode (see Figure 1). Cropping to small areas $\left(\sim 1 \mathrm{~m}^{2}\right.$, compared to initially $\sim 200$ $\mathrm{m}^{2}$ for the complete gravel bar) allowed interpolation of SfM-derived elevations onto fine grids, here we used $1 \mathrm{~mm}$ spacing. This way, small-scale topography such as sediment grains and bed undulations (bedforms) present on the gravel bar are revealed by the DEMs (Figure 6).

Figure 6 shows an example of comparison between SfM and stereo photogrammetry, based on patch 11. Visually, both SfM and stereo photogrammetry DEMs are able to represent the surface humps and hollows, as well as the largest gravels (Figures 6A and 6B). Further, the two DEMs are contained in the same elevation range and none present obvious outliers. However, the higher resolution afforded by stereo photogrammetry in this study (raw point spacing of $0.19 \mathrm{~mm}$ compared to $3.3 \mathrm{~mm}$ with SfM) greatly improves surface representation (such as the shape of sediment grains and fine details) and the overall DEM quality. Figure 6C shows the DoD resulting from 
subtracting the stereo photogrammetry DEM from the SfM DEM. Colour coding was adapted to enable easy identification of large errors (here absolute elevation differences above $5 \mathrm{~mm}$ ). There are few (large) errors in the centre of the DoD. However, errors become more frequent as we move towards the DEM boundaries. Particularly, it shows alternating negative and positive errors on the right side of the $\mathrm{DoD}$, and the inverse sequence on the left side. This indicates a scale difference between the two DEMs, whereby the SfM DEM is stretched horizontally compared to stereo photogrammetry. Quantitatively, SfM DEM errors are represented by MUE and SDE of $1.52 \mathrm{~mm}$ and 2.02 mm, respectively (see Table 2). Inspection of the DoD (Figure 6C) shows no obvious curvature in the SfM DEM in that no significant trend was identified. This echoes our previous assertion that the photo-DEM collected with SfM is free of large non-linear distortions, and thus was adequate as benchmark for evaluating the video-DEM curvature (Figure 5).
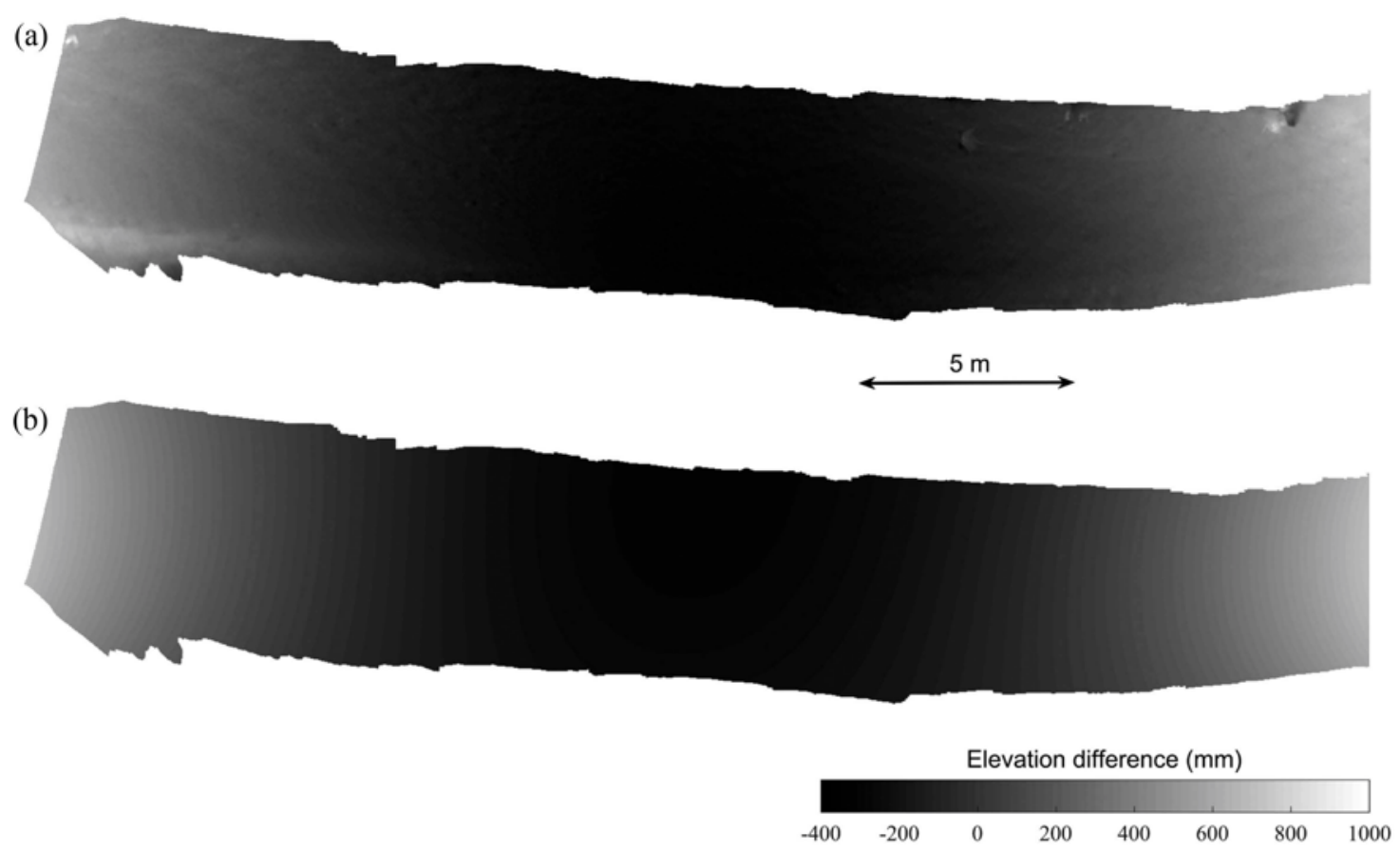

Figure 5. (a) DEM of difference (DoD) between SfM measurements obtained using photo- and videomode, respectively; and (b) curvature identified in DoD. 
Similarly to the example of patch 11 presented in Figure 6, SfM was compared with stereo photogrammetry for all 11 patches contained in the photo-DEM, and SfM errors were quantified in the case DEMs were reconstructed using 3 and $10 \mathrm{CPs}$, respectively (Table 2). The reference patch used for producing CPs in this part of the analysis is patch 9. Two important observations can be done in Table 2. First, DEM quality changes minimally when $\mathrm{CP}$ quantity is increased from 3 to 10 . DEM accuracy (here represented by MUE and SDE after comparison with stereo photogrammetry) only improves for 4 out of the 11 patches. For the other 7 patches, DEM accuracy is better in the case scaling was provided using 3 CPs (Table 2). Overall (i.e. after averaging errors across all patches), DEM accuracy is slightly $(\sim 3 \%)$ better for $3 \mathrm{CPs}(\mu=2.51 \mathrm{~mm}$ and $\sigma$ $=0.67 \mathrm{~mm}, \mathrm{n}=11)$ compared to using $10 \mathrm{CPs}(\mu=2.59 \mathrm{~mm}$ and $\sigma=0.65 \mathrm{~mm})$. Table 2 also presents the rotation angle applied during DEM alignment. Mutual alignment between SfM and stereo photogrammetry DEMs required varying rotation angles depending on the patch location (Table 2, Figure 7). However, consistent angles were obtained regardless of CP quantity, with a maximum difference of 1 degree (which corresponds to the angular resolution during alignment). This is indicative of both a precise alignment strategy, ensuring reliable error statistics after comparison with stereo photogrammetry, and consistency between SfM DEMs obtained using 3 and 10 CPs, respectively. Not surprisingly, there is no rotation necessary (i.e. angle $=0$ degree) to align SfM and stereo photogrammetry in the case of patch 9, as it is the reference patch.

Second observation in Table 2, DEM accuracy is dependent on the patch location, with observed variations in MUE ranging from $1.52 \mathrm{~mm}$ (patch 12) to $3.48 \mathrm{~mm}$ (patch 3) in the case of 3 CPs. Different reasons, investigated below, may be referred to for explaining this variability in DEM accuracy across the gravel bar. There is the possibility that bar-scale variability in morphology and bed structure (e.g., variations due to sediment 
size, bed elevations, and vegetation across the bar) explains varying DEM accuracy, as surface texture and roughness are known influences on matching performance and occlusions (e.g., Bertin et al. 2015; Lane 2000). There is also the possibility that the location of the reference patch influences SfM dimension scaling, with the eventuality that DEM accuracy is optimum at or near the reference patch and degraded otherwise. The fact that DEM accuracy variability across patches appears relatively consistent for measurements performed both with 3 and 10 CPs (Table 2) tends to support the two possibilities invoked.
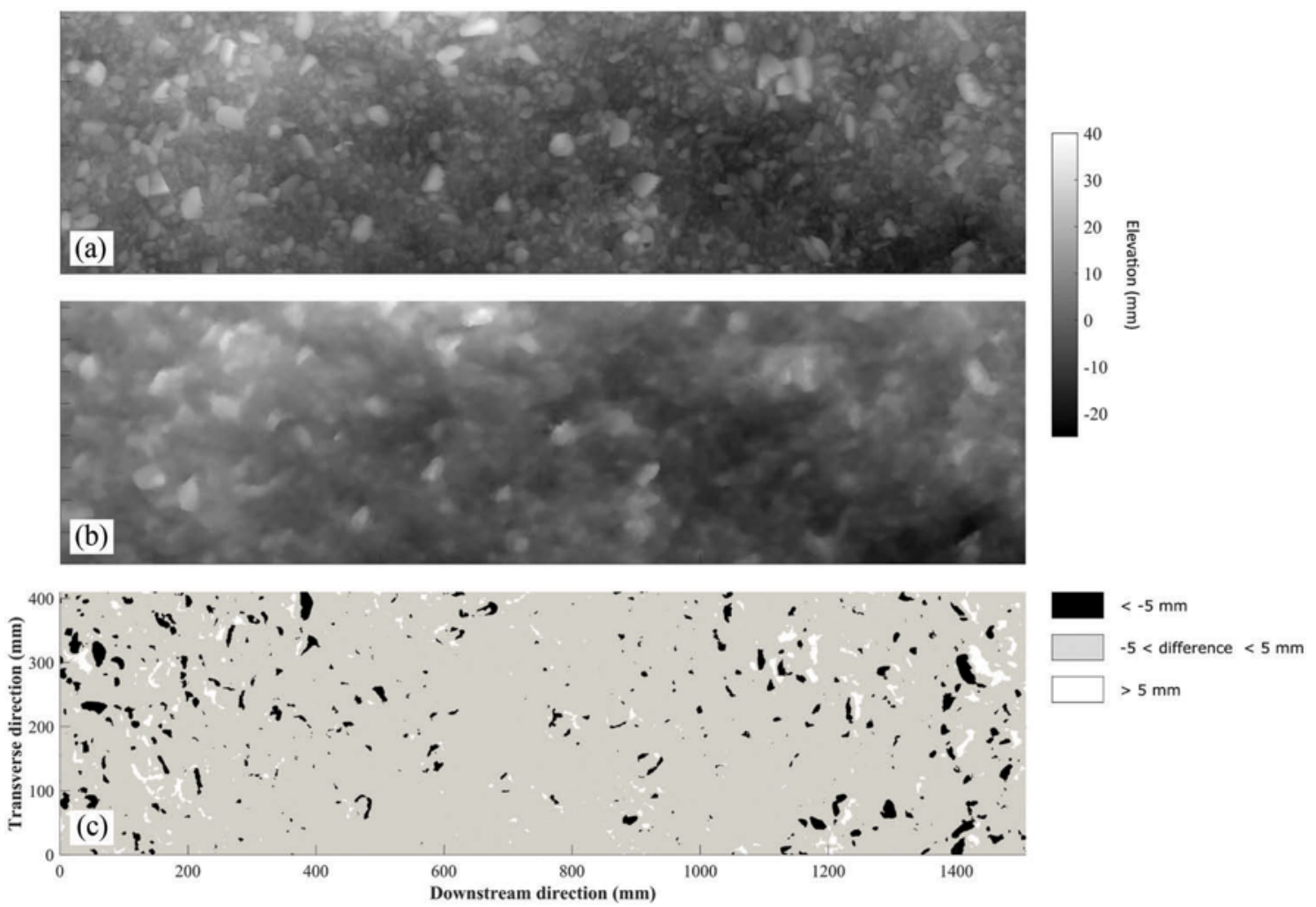

Figure 6. SfM comparison with stereophotogrammetry, an example of patch 11, showing (a) the DEM obtained with stereophotogrammetry (raw horizontal resolution of 0.19 $\mathrm{mm}$ ); (b) the SfM DEM using photo mode (raw horizontal resolution of $3.3 \mathrm{~mm}$ ); and (c) DoD. All DEMs were interpolated onto grids with $1 \mathrm{~mm}$ spacing before comparison. The SfM DEM used patch 9 as the reference patch and 3 CPs. 
Table 2. Comparison between SfM and stereophotogrammetry, for 3 and 10 CPs used for SfMdimension scaling (reference patch is patch 9). MUE and SDE are mean unsigned and standard deviation of error, respectively, obtained after point-by-point comparison between mutually aligned $\mathrm{SfM}$ and stereo photogrammetry DEMs. Grid spacing is $1 \mathrm{~mm}$. Rotation corresponds to the rotation angle applied to stereo- photogrammetry DEMs during alignment with SfM DEMs (+ ve values are for clockwise rotations, - ve values for anti-clockwise). Angular resolution during alignment is $1^{\circ}$. Distance is the rounded distance to the reference patch, measured between the two patch centres.

\begin{tabular}{|c|c|c|c|c|c|c|c|}
\hline & \multicolumn{2}{|c|}{ MUE (mm) } & \multicolumn{2}{|c|}{$\mathrm{SDE}(\mathrm{mm})$} & \multicolumn{2}{|c|}{ Rotation ( $\left.{ }^{\circ}\right)$} & \multirow[b]{2}{*}{ Distance $(\mathrm{m}$} \\
\hline & $3 \mathrm{CPs}$ & $10 \mathrm{CPs}$ & $3 \mathrm{CPs}$ & $10 \mathrm{CPs}$ & $3 \mathrm{CPs}$ & $10 \mathrm{CPs}$ & \\
\hline Patch3 & 3.24 & 2.97 & 4.37 & 4.02 & 3 & 2 & 11 \\
\hline Patch4 & 3.24 & 3.21 & 4.75 & 4.60 & 3 & 2 & 9 \\
\hline Patch5 & 3.48 & 3.41 & 4.86 & 4.70 & 3 & 3 & 5 \\
\hline Patch7 & 2.79 & 3.16 & 3.87 & 4.34 & -2 & -2 & 4 \\
\hline Patch 8 & 1.72 & 1.71 & 2.77 & 2.70 & -3 & -3 & 2 \\
\hline Patch 9 & 2.09 & 2.21 & 2.76 & 2.83 & 0 & 0 & 0 \\
\hline Patch 10 & 2.91 & 3.16 & 4.18 & 4.45 & 0 & 0 & 2 \\
\hline Patch11 & 1.93 & 2.02 & 2.64 & 2.77 & 0 & 0 & 5 \\
\hline Patch12 & 1.52 & 1.59 & 2.02 & 2.17 & -7 & -8 & 7 \\
\hline Patch 13 & 2.45 & 2.68 & 3.55 & 3.80 & -7 & -6 & 7 \\
\hline Patch14 & 2.20 & 2.32 & 3.54 & 3.64 & -4 & -5 & 9 \\
\hline Mean & 2.51 & 2.59 & 3.57 & 3.64 & & & \\
\hline SD & 0.67 & 0.65 & 0.93 & 0.88 & & & \\
\hline
\end{tabular}

\subsection{Effect of the location of the reference patch}

Figure 7 shows the effect of varying the reference patch, used for providing scaled coordinates for SfM measurements, on the distribution of DEM errors, obtained after comparison with stereo photogrammetry. The gravel bar is split into three sections every 10 meters, dividing it into bar head, bar centre and bar tail, as used in previous studies (Rice and Church 2010). Visually, using patch 3 or patch 9 resulted in different spatial distributions of DEM errors. One can see that DEM errors are fewer at or near the location of the reference patch, and are generally higher elsewhere. For instance, Figure 7A shows that choosing patch 9 as the reference patch resulted in DEM errors higher on the upstream side of the bar, whereas the case of patch 3 (Figure 7B) shows few DEM errors at the bar head where patch 3 is located yet more errors at the bar centre and bar tail.

Table 3 confirms differences in the distribution of errors between SfM DEMs scaled with patch 3 and patch 9, respectively. If using patch 3, which is located upstream of the bar, SfM measurements show better agreement with reference measurements 
produced with stereo photogrammetry for those comparisons at the bar head (MUE $=2.08$ $\mathrm{mm}$ ), whilst errors increase in other parts of the gravel bar. If using patch 9, which is located at the bar centre, measurement accuracy is worse at the bar head (MUE $=3.24$ $\mathrm{mm}$ ), and improves for both the bar centre and the bar tail. If looking at the rotation angle during alignment with stereo photogrammetry, one can see varying angles depending on the reference patch used. Careful inspection of Table 3, which presents the rotation angle averaged for each bar location (i.e. bar head, centre and tail), shows however a same pattern in rotation angle regardless of the reference patch. Using patch 3 meant there is no need for rotation correction at the bar head (rotation angle $=0$ ), but patches at the bar centre and bar tail needed rotation in average of -3 and -8 degrees, respectively. Similarly, using patch 9 meant there is no need for rotation correction at the bar centre, but 3 and 5 degrees corrections were needed at the bar head and bar tail, respectively. Altogether, this shows that the overall bar structure measured with SfM is consistent regardless of the reference patch used for providing a scaled reference coordinate system.

Table 3. Error distribution across three bar sub-sections: bar head (patches 3 and 4), bar centre (patches 5, 7, 8, 9, 10 and 11) and bar tail (patches 12, 13 and 14), for SfM DEMs produced using either patch 3 or patch 9 as the reference. Error statistics and rotation angles were averaged over all patches contained in the sub-sections.

\begin{tabular}{llccc}
\hline Location & Reference for scaling & MUE $(\mathrm{mm})$ & SDE $(\mathrm{mm})$ & Rotation $\left(^{\circ}\right)$ \\
\hline \multirow{2}{*}{ Bar head } & Patch 3 & 2.08 & 2.94 & 0 \\
& Patch 9 & 3.24 & 4.56 & 3 \\
\multirow{3}{*}{ Bar centre } & Patch 3 & 3.30 & 4.54 & -3 \\
& & & & \\
\multirow{2}{*}{ Bar tail } & Patch 9 & 2.60 & 3.69 & 0 \\
& Patch 3 & 2.92 & 4.34 & -8 \\
\hline
\end{tabular}




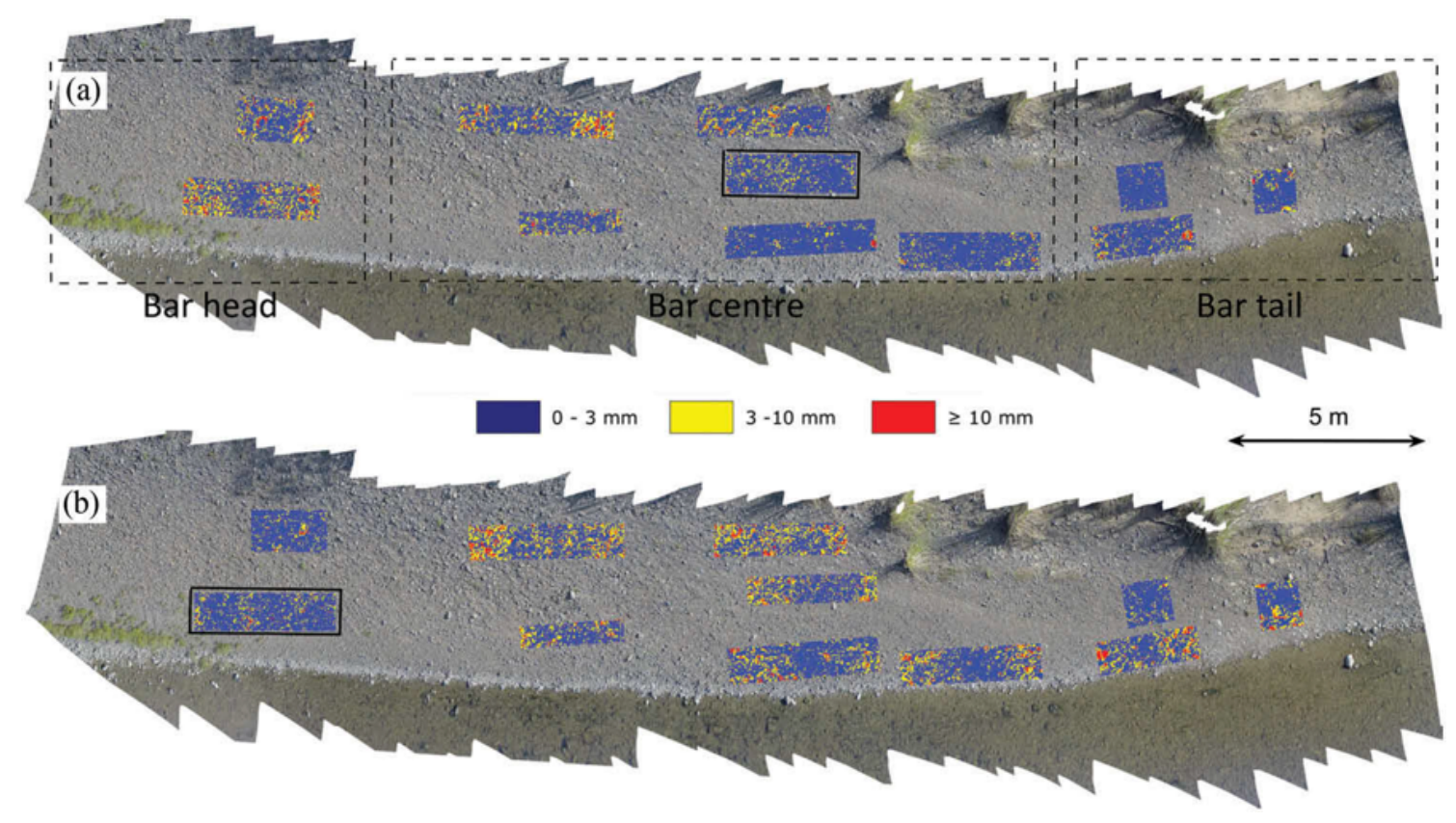

Figure 7. Distribution of absolute DEM errors produced after comparison with 11 'truth DEMs' collected independently with stereophotogrammetry, for (a) the SfM-DEM scaled using patch 9 as the reference; and (b) the SfM-DEM scaled using patch 3 as the reference. For easy interpretation, error maps are magnified $\times 1.2$ and the patch used as a reference is framed. Dashed rectangles show the three bar sub-sections (bar head, bar centre and bar tail) used for error distribution analysis.

In Figure 8, we investigate possible reasons explaining different spatial error distributions resulting from using different reference patches. Using CloudCompare (Girardeau-Montaut 2011), SfM DEMs produced using patch 3 and patch 9, respectively, were found to differ by a horizontal scaling factor of 0.96 . Before scale correction (Figure 8A), DEM comparison shows the occurrence of large differences (here represented by errors above $10 \mathrm{~mm}$ ) throughout the bar. Quantitatively, differences are characterised by MUE $=11.2 \mathrm{~mm}, \mathrm{SDE}=18.4 \mathrm{~mm}$ and a maximum absolute difference of $504.4 \mathrm{~mm}$. After scale correction (Figure 8B), differences between DEMs are significantly reduced, indicated by $\mathrm{MUE}=5.5 \mathrm{~mm}, \mathrm{SDE}=10.0 \mathrm{~mm}$ and a maximum absolute difference of $355.3 \mathrm{~mm}$. Visually, this is shown by a large portion $(\sim 35 \%)$ of the gravel bar with 
measurement errors (i.e. differences between DEMs) below $3 \mathrm{~mm}$ and nearly $90 \%$ of the bar with errors below $10 \mathrm{~mm}$. Furthermore, no particular trend was detected in the DoD, which again, supports the absence of curvature in the photo-DEMs.

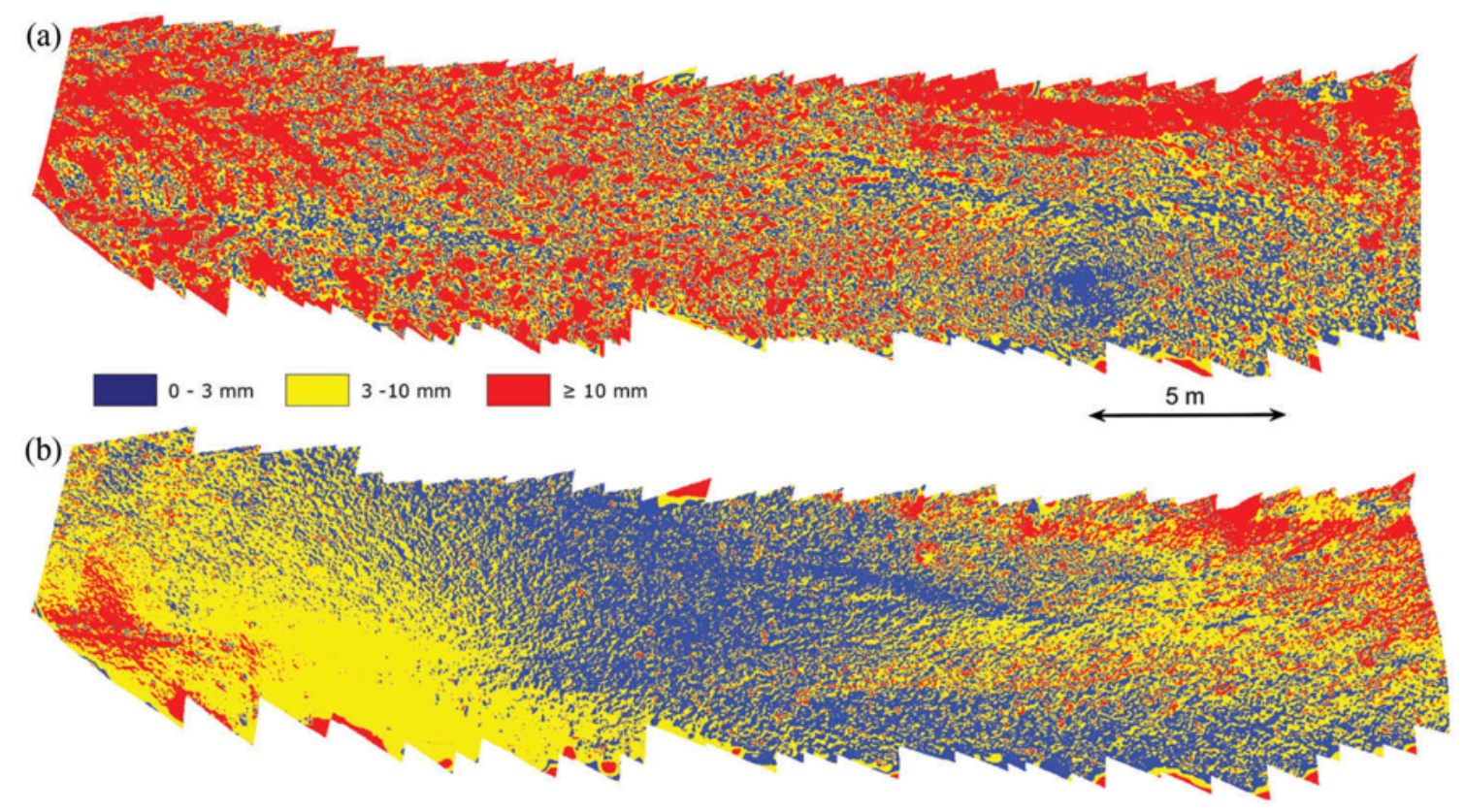

Figure 8. DEM of absolute difference (DoAD) between SfM DEMs obtained using patch 3 and patch 9 as reference patches, (a) before; and (b) after correcting for horizontal scale differences. Scale correction was determined using CloudCompare (Girardeau-Montaut 2011), showing a factor equal to 0.96 correctly accounts for differences in horizontal scaling between DEMs.

\subsection{Error characterisation in application to a gravel bar}

Figure 9 compares SfM DEM errors, resulting from comparisons with stereo photogrammetry at 11 patches (Table 2 ) with landmark characteristics, such as surface irregularity, represented by the standard deviation of bed elevation $\left(\sigma_{\mathrm{Z}}\right)$, and surface sediment size, represented by the median grain size $\left(D_{50}\right) . \sigma_{Z}$ was determined for each patch measured with stereo photogrammetry, and detrended to remove bed slope influence. $\mathrm{D}_{50}$ was determined using a single Nikon D5100 photograph for each patch and Basegrain, a Matlab toolbox for sediment size analysis (Detert and Weitbrecht 2012). 
For this analysis, SfM DEMs were produced using photo mode, patch 9 as the reference and 3 CPs. The findings also apply to SfM DEMs reconstructed differently, for instance using video mode, $10 \mathrm{CPs}$ and patch 3 used as the reference patch.
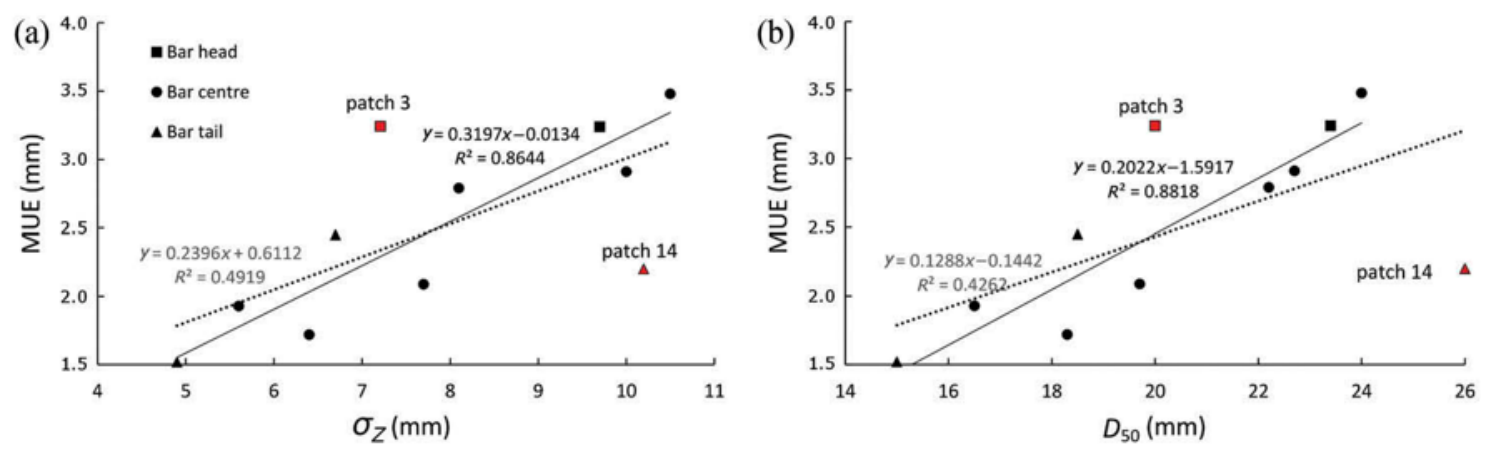

Figure 9. Relationships between SfM measurement error - represented by MUE and determined by comparing SfM (photo mode, patch 9 and 3 CPs) with stereophotogrammetry - and standard deviation of bed elevations $(\sigma Z)$ and surface sediment size (D50) at 11 patches. Dashed lines represent the linear relationships minimising the sum of the squared distances between all data points $(n=11)$ and the fitted lines, whilst continuous lines omit patches 3 and 14 from the relations $(n=9)$.

Figure 9 shows positive linear relations between surface irregularity, sediment size and measurement errors. SfM DEM accuracy is higher for smooth patches comprising small sediment, whereas DEM errors increase (i.e. accuracy lessens) for irregular patches comprising coarser sediment. Two patches (patch 3 and patch 14) are responsible for most of the scatter observed in Figure 9, as exemplified by a two-fold increase in $\mathrm{R}^{2}$ after removing the two eccentric points. Patch 14 is the patch with the largest $\mathrm{D}_{50}$ we measured in this study. However, visual examination of patch 14 reveals two large gravels onto an otherwise smooth surface made of smaller sediment, which may explain a smaller measurement error than predicted for this patch. 


\section{Discussion}

The workflow presented has allowed collection of spatially continuous topographic data over a complete river bar (surface coverage $\sim 200 \mathrm{~m}^{2}$ ), exposed at the time of measurement, using a combination of classic stereo photogrammetry and SfM processing methods. We obtained accurate DEMs at both bar and patch scale, for which georeferencing to an independent coordinate system was not necessary.

For allowing the effective use of fish-eye lens action cameras such as GoPro Hero 3 for SfM, and for obviating the need for physical GCPs disposed on the field site, we have implemented two adaptations to the ordinary workflow embodied in Agisoft: (1) doing camera calibration independently from Agisoft; and (2) using independent measurements performed with stereo photogrammetry to provide scaled coordinates during DEM reconstruction.

\subsection{SfM workflow for GoPro Hero 3}

Using high-distortion images, such as those recorded with a GoPro Hero 3, full DEM reconstruction using SfM is limited to a few recent studies, with previous work generally mentioning lower DEM quality compared to what is achievable using higher-quality DSLRs (Carbonneau and Dietrich 2017; Ingwer et al. 2015; Marteau et al. 2017; Smith, Carrivick, and Quincey 2015). Two factors appear to directly contribute to DEM quality: image distortion and image resolution.

In James and Robson (2012), it was suggested using DSLR cameras and fixedfocus lenses for the sake of effective image distortion modelling during calibration. However, a more recent study showed that distortion modelling with high-order polynomials can help achieve high-quality DEMs with GoPro Hero 3 (e.g., $\sim 0.015 \mathrm{~m}$ root-mean square error (RMSE) after comparison with surveyed points at a distance of 5 
m (Marteau et al. 2017)). As a comparison, MUE below $0.004 \mathrm{~m}$ (Table 2) was achieved across the bar from a vertical distance $\sim 2 \mathrm{~m}$ using GoPro Hero 3 in this study. In spite of difficulties comparing results between the two studies, partly due to differences in camera height, DEM grid spacing and accuracy evaluation methods used, both studies indicate the possibility of using GoPro Hero 3 for accurate DEM collection with SfM.

In any case, special attention must be given to camera calibration, such as using high-order distortion models and increasing the quantity of calibration images. SfM processing of both original and undistorted images collected with a GoPro Hero 3 was compared in Balletti et al. (2014). They showed that image un-distortion improved DEM accuracy two-fold. Marteau et al. (2017) used the calibration method included in Agisoft (called 'Agisoft Lens') for calibrating a GoPro Hero 3, whereas Zhang calibration was used herein. Both methods used a planar chequerboard and included the $k 3$ and $k 4$ distortion coefficients to account for tangential distortion, which is advised when using fish-eye lenses for SfM (Marteau et al. 2017). In terms of image quantity, Marteau et al. (2017) used five calibration images compared to 29 images in this study, which would tend to support advantages to using Agisoft Lens. Performing calibration and image undistortion outside Agisoft contributes to the originality of our workflow. The decision was motivated by the works of Harwin, Lucieer, and Osborn (2015) who compared Agisoft Lens with other calibration methods and found that calibration performed with Agisoft provided the worse results ( five times less accurate than the other methods). Further work comparing available calibration methods in application to fish-eye lens cameras such as GoPro is necessary, and would ultimately help researchers decide calibration requirements for their study.

Previous research consistently preferred data acquisition with GoPro using photo mode, due to the larger image format and resulting denser point cloud (Harwin, Lucieer, 
and Osborn 2015; James et al. 2017; Marteau et al. 2017). In our study, point cloud resolutions of $2.9 \mathrm{~mm}$ and $3.3 \mathrm{~mm}$ were achieved with video frames and photos, respectively. The better resolution achieved with video mode was nevertheless balanced by the significantly larger number of video frames processed (317 video frames compared to 32 photos). Comparison of DEMs reconstructed using video frames and photos, respectively, has indicated the presence of convex curvature in the video-DEM. We believe the curvature of the video-DEM is the result of a very large image overlap ( $95 \%)$, together with a near vertical camera orientation, which made for highly redundant information processed with $\mathrm{SfM}$, and hindered convergence of the optimised camera parameters.

The affordable price, the convenient operation (e.g., when combined with a pole or drone and controlled with mobile phone or iPad) and the large FoV, are all tangible reasons that motivated us using GoPro Hero 3 in this study. While GoPro Hero 3 camera is perhaps not the best argument in terms of data quality, the advantages mentioned above, together with reasonable resolution ( $\sim 3 \mathrm{~mm}$ point spacing) and accuracy ( $\sim 4 \mathrm{~mm}$ MUE) achieved in this study, clearly demonstrate the potential of using GoPro Hero 3 and SfM in the field.

\subsection{Feasibility of camera only topographic remote sensing}

Another originality of the workflow presented is the use of 'control points' extracted from an independent DEM collected with stereo photogrammetry, providing scaled coordinates and allowing quantitative analysis with SfM. We believe no other research has implemented this method before. Traditional methods requiring (physical) GCPs to be organised on the study site, geo-referenced using independent measurements (e.g., total station, or GPS), suffer from both time and resource demands, for example three hours to arrange GCPs and around $\$ 2,000$ for the cost of a total station (Marteau et al. 
2017). Improving time and cost efficiency, for example by decreasing the quantity of GCPs and/or using low-cost equipment (e.g., low precision GPS module), has generally come at the cost of degraded DEM accuracy.

In contrast, CPs acquired with stereo photogrammetry were used in this study. The latter provided horizontal and vertical resolutions of $0.19 \mathrm{~mm}$ and $0.47 \mathrm{~mm}$, respectively, which surpass other studies using total stations, with reported GCP referencing accuracy of $5 \mathrm{~mm}$ and $10 \mathrm{~mm}$, in the horizontal and vertical direction, respectively (d'Oleire-Oltmanns et al. 2012), or $10 \mathrm{~mm}$ vertical accuracy reported by Morgan, Brogan, and Nelson (2017) who used TLS at a distance of approximately $10 \mathrm{~m}$. The whole process of collecting DEMs over 14 gravel patches took approximately one hour. Besides providing benchmarks for scaling and assessing SfM measurements, these data allow for detailed geomorphic analysis of sections of the bar otherwise not possible at the scale covered with SfM.

Although previous work generally reported that DEM quality improves with GCP quantity, this was not verified in our work. Global DEM accuracy over the gravel bar was found to be characterised by MUE of $2.51 \mathrm{~mm}(\sigma=0.67 \mathrm{~mm})$ in the case of 3 CPs (Table 2). Using 10 CPs provided slightly worse results, whereby MUE increased by $\sim 3 \%$. Although further work is necessary to confirm this finding, our results indicate that $\mathrm{CP}$ quality (especially referencing accuracy) is more important than CP quantity.

This raises the question of whether the process of using CPs and establishing a point-point correspondence between datasets used in the present study is preferable, compared to using external methods such as CloudCompare (Girardeau-Montaut 2011), which would allow using a complete stereo photogrammetry DEM to provide scaled coordinates for SfM after mutual alignment. This method was used previously by Morgan, Brogan, and Nelson (2017), whereby SfM DEMs collected in a laboratory 
environment were scaled using TLS measurements. The method has the advantage of obviating the need for manual selection of CPs, however requires a more elaborate and disconnected workflow. In contrast, the process of using CPs in this study was similar to using GCPs, and allowed simple processing in Agisoft.

Using CPs extracted from an independent DEM to scale SfM results is in essence comparable to using scale bars or ground truth 3D objects. Both methods allow to collect high-quality topographic surface data by only using cameras, and alleviate the need for GCPs and associated survey equipment. However, using a DEM as control is less intrusive, and allows measurement of the whole terrain without obstructions due to scale bars or other known objects. Besides, a DEM provides many control points at different distances and elevations, and a DEM has fewer size constraints than a 3D ground truth object, thus is more efficient and effective to use in the field.

The reference DEM providing the CPs was produced with stereo photogrammetry in our work. Although other techniques, such as laser scanning, may be used, using stereo photogrammetry has the advantage of reducing the cost, facilitating transport and usage in difficult terrains, as well as improving sampling resolution important for $\mathrm{CP}$ accuracy. Furthermore, we found texture information to be helpful for extracting precise CPs (Figure 3), something which may not be possible if using TLS.

\section{Conclusion}

Recent developments in SfM have eased the difficulty with which topographic data can be collected. However, the need to have physical GCPs deployed on the study site and measured independently still hinders the full potential of SfM in application to remote terrains. This work evaluates a combined approach of SfM and stereo photogrammetry for studying gravel bars. 
We present the application of SfM using Agisoft PhotoScan and GoPro Hero 3, in tandem with stereo photogrammetry using two Nikon D5100s, to measure an exposed gravel bar. We show how stereo photogrammetry provides accurate sub-millimetre resolution DEMs, enabling detailed textural and structural riverbed characterization, from which control points (CPs) were extracted to provide scaled coordinates necessary for quantitative measurements using SfM. Our evaluation shows that over an area of 11 gravel patches, the differences between SfM and stereo photogrammetry are consistently small, in the order of a few millimetres. However, the location of the reference stereo photogrammetry DEM used for providing the CPs was found to have a larger influence on SfM DEM quality. Notably, fewer errors were measured at or near the reference patch. Away from the reference patch, errors generally increased, which is indicative that the location of the reference patch influences SfM DEM accuracy globally. Differences in the spatial distribution of SfM errors were found to arise from minor (scaling factor of 0.96) scale differences between DEMs produced with varied reference patches. Attempts to reconstruct DEMs using video frames recorded with GoPro Hero 3 provided the least quality results, which is attributed to the smaller image format, and hence coarser point spacing in the obtained point cloud, which eventually results in a convex curvature when image overlap was increased.

Our results show that it is possible to collect high-quality and properly scaled topographic field data using only cameras. The proposed workflow reduces the complexity generally encountered by using traditional GCPs, as well as reduces time and resource demands associated with deploying GCPs and necessary independent georeferencing. Given our study's results, we expect digital photogrammetry to continue gaining popularity for field surveying, as it encompasses a light-weight, low-cost and versatile, and as we show, accurate technique. 


\section{Acknowledgements}

The authors thank Jane Groom for her assistance in the collection of field data, and Jon

Tunnicliffe for discussion. The study was partly funded by the Marsden Fund (Grant No.

UOA1412), administered by the Royal Society of New Zealand.

\section{References}

Balletti, C., F. Guerra, V. Tsioukas, and P. Vernier. 2014. "Calibration of Action Cameras for Photogrammetric Purposes." Sensors 14 (9):17471-90. doi: 10.3390/s140917471.

Bertin, S., and H. Friedrich. 2014. "Measurement of Gravel-bed Topography: Evaluation Study Applying Statistical Roughness Analysis." Journal of Hydraulic Engineering 140 (3):269-79. doi: 10.1061/(ASCE)HY.1943-7900.0000823. 2016. "Field Application of Close-range Digital Photogrammetry (CRDP) for Grain-scale Fluvial Morphology Studies." Earth Surface Processes and Landforms 41:1358-69. doi: 10.1002/esp.3906.

Bertin, S., H. Friedrich, and P. Delmas. 2016. "A Merging Solution for Close-Range DEMs to Optimize Surface Coverage and Measurement Resolution." Photogrammetric Engineering \& Remote Sensing 82 (1):31-40. doi: 10.14358/pers.83.1.31.

Bertin, S., H. Friedrich, P. Delmas, and E. Chan. 2013. "On the Use of Close-range Digital Stereo-photogrammetry to Measure Gravel-bed Topography in a Laboratory Environment." Proc. 35th IAHR Congress:unpaginated CD-ROM.

Bertin, S., H. Friedrich, P. Delmas, E. Chan, and G. Gimel'farb. 2015. "Digital Stereo Photogrammetry for Grain-scale Monitoring of Fluvial Surfaces: Error Evaluation and Workflow Optimisation." ISPRS Journal of Photogrammetry and Remote Sensing 101 (0):193-208. doi: 10.1016/j.isprsjprs.2014.12.019.

Bird, S., D. Hogan, and J. Schwab. 2010. "Photogrammetric Monitoring of Small Streams under a Riparian Forest Canopy." Earth Surface Processes and Landforms 35 (8):952-70. doi: 10.1002/esp.2001.

Bouguet, J-Y. 2010. "Camera calibration toolbox for Matlab." http://www.vision.caltech.edu/bouguetj/calib_doc/Caltech, Pasadena, Calif.

Brunier, G., J. Fleury, E. J. Anthony, A. Gardel, and P. Dussouillez. 2016. "Close-range Airborne Structure-from-Motion Photogrammetry for High-resolution Beach Morphometric Surveys: Examples from an Embayed Rotating Beach." Geomorphology 261:76-88. doi: 10.1016/j.geomorph.2016.02.025.

Buffington, J. M., and D. R. Montgomery. 1999. "A Procedure for Classifying Textural Facies in Gravel-bed Rivers." Water Resources Research 35 (6):1903-14. doi: 10.1029/1999wr900041.

Carbonneau, P. E. , S. N. Lane, and N. E. Bergeron. 2003. "Cost-effective Non-metric Close-range Digital Photogrammetry and its Application to a Study of Coarse Gravel River Beds." International Journal of Remote Sensing 24 (14):2837-54. doi: 10.1080/01431160110108364.

Carbonneau, P. E., and J. T. Dietrich. 2017. "Cost-effective Non-metric Photogrammetry from Consumer-grade sUAS: Implications for Direct Georeferencing of Structure 
from Motion Photogrammetry." Earth Surface Processes and Landforms 42 (3):473-86. doi: 10.1002/esp.4012

Carrivick, J. L., M. W. Smith, J. Q. Duncan, and S. J. Carver. 2013. "Developments in Budget Remote Sensing for the Geosciences." Geology Today 29 (4):138-43. doi: 10.1111/gto.12015.

Chandler, J. H., J. G. Fryer, and A. Jack. 2005. "Metric Capabilities of Low-cost Digital Cameras for Close Range Surface Measurement." Photogrammetric Record 20 (109):12-26. doi: 10.1111/j.1477-9730.2005.00302.x.

Chandler, J., K. Shiono, P. Rameshwaran, and S. Lane. 2001. "Measuring Flume Surfaces for Hydraulics Research Using a Kodak DCS460." The Photogrammetric Record 17 (97):39-61. doi: 10.1111/0031-868X.00167.

Church, M. A., D. G. McLean, and J. F. Wolcott. 1987. River bed gravel: sampling and analysis. Edited by C.R. Thorne, Bathurst, J.C., Hey, R.D., Sediment Transport in Gravel-bed River: Wiley, Chichester.

d'Oleire-Oltmanns, S., I. Marzolff, K. D. Peter, and J. B. Ries. 2012. "Unmanned Aerial Vehicle (UAV) for Monitoring Soil Erosion in Morocco." Remote Sensing 4 (11):3390-416. doi: 10.3390/rs4113390.

De Souza, C. H. W., R. A. C. Lamparelli, J. V. Rocha, and P. S. G. Magalhães. 2017. "Height Estimation of Sugarcane Using an Unmanned Aerial System (UAS) Based on Structure from Motion (SfM) Point Clouds." International Journal of Remote Sensing 38 (8-10):2218-30. doi: 10.1080/01431161.2017.1285082.

Detert, M., and V. Weitbrecht. 2012. "Automatic Object Detection to Analyze the Geometry of Gravel Grains." Proc. RiverFlow 2012:595-600.

Entwistle, N. S., and I. C. Fuller. 2009. "Terrestrial Laser Scanning to Derive Surface Grain Size Facies Character of Gravel Bars." In Laser Scanning for the Environmental Sciences, 102-14. Wiley-Blackwell.

Fonstad, M. A., J. T. Dietrich, B. C. Courville, J. L. Jensen, and P. E. Carbonneau. 2013. "Topographic Structure from Motion: A new Development in Photogrammetric Measurement." Earth Surface Processes and Landforms 38 (4):421-30. doi: 10.1002/esp.3366.

Gimel'farb, G. 2002. "Probabilistic Regularisation and Symmetry in Binocular Dynamic Programming Stereo." Pattern Recognition Letters 23 (4):431-42. doi: 10.1016/s0167-8655(01)00175-1.

Girardeau-Montaut, D. 2011. "CloudCompare (Version 2.6.2): 3D Point Cloud and Mesh Processing Software." http://www.danielgm.net/cc/.

Groom, J., S. Bertin, and H. Friedrich. 2018. "Assessing Intra-Bar Variations in Grain Roughness Using Close-Range Photogrammetry." Journal of Sedimentary Research 88 (5):555-67. doi: 10.2110/jsr.2018.30.

Harwin, S., A. Lucieer, and J. Osborn. 2015. "The Impact of the Calibration Method on the Accuracy of Point Clouds Derived Using Unmanned Aerial Vehicle MultiView Stereopsis." Remote Sensing 7 (9):11933-53. doi: 10.3390/rs70911933.

Hassan, M. A. 2005. "Characteristics of Gravel Bars in Ephemeral Streams." Journal of Sedimentary Research 75 (1):29-42. doi: 10.2110/jsr.2005.004.

Hauer, C., G. Unfer, P. Holzapfel, M. Haimann, and H. Habersack. 2014. "Impact of Channel Bar Form and Grain Size Variability on Estimated Stranding Risk of Juvenile Brown Trout during Hydropeaking." Earth Surface Processes and Landforms 39 (12):1622-41. doi: 10.1002/esp.3552.

Heritage, G. L., and D. J. Milan. 2009. "Terrestrial Laser Scanning of Grain Roughness in a Gravel-bed River." Geomorphology 113 (1-2):4-11. doi: 10.1016/j.geomorph.2009.03.021. 
Hodge, R., J. Brasington, and K. Richards. 2009. "Analysing Laser Scanned Digital Terrain Models of Ggravel Bed Surfaces: Linking Morphology to Sediment Transport Processes and Hydraulics." Sedimentology 56 (7):2024-43. doi: 10.1111/j.1365-3091.2009.01068.x.

Ingwer, P., F. Gassen, S. Püst, M. Duhn, M. Schälicke, K. Müller, H. Ruhm, J. Rettig, E. Hasche, and A. Fischer. 2015. "Practical Usefulness of Structure from Motion (SfM) Point Clouds Obtained from Different Consumer Cameras." SPIE/IS\&T Electronic Imaging:941102-11. doi: 10.1117/12.2074892.

James, M. R., and S. Robson. 2012. "Straightforward Reconstruction of 3D Surfaces and Topography with a Camera: Accuracy and Geoscience Application." Journal of Geophysical Research: Earth Surface 117 (F3). doi: 10.1029/2011JF002289.

_ 2014. "Sequential Digital Elevation Models of Active Lava Flows from Groundbased Stereo Time-lapse Imagery." ISPRS Journal of Photogrammetry and Remote Sensing 97:160-70. doi: 10.1016/j.isprsjprs.2014.08.011.

James, M. R., S. Robson, S. d'Oleire-Oltmanns, and U. Niethammer. 2017. "Optimising UAV Topographic Surveys Processed with Structure-from-Motion: Ground Control Quality, Quantity and Bundle Adjustment." Geomorphology 280:51-66. doi: 10.1016/j.geomorph.2016.11.021.

Javernick, L., J. Brasington, and B. Caruso. 2014. "Modeling the Topography of Shallow Braided Rivers using Structure-from-Motion Photogrammetry." Geomorphology 213:166-82. doi: 10.1016/j.geomorph.2014.01.006.

Lane, S. N. 2000. "The Measurement of River Channel Morphology Using Digital Photogrammetry." The Photogrammetric Record 16 (96):937-61. doi: 10.1111/0031-868x.00159.

Lane, S. N., R. M. Westaway, and M. D. Hicks. 2003. "Estimation of Erosion and Deposition Volumes in a Large, Gravel-bed, Braided River Using Synoptic Remote Sensing." Earth Surface Processes and Landforms 28 (3):249-71. doi: 10.1002/esp.483.

Marteau, B., D. Vericat, C. Gibbins, R. J. Batalla, and D. R. Green. 2017. "Application of Structure-from-Motion Photogrammetry to River Restoration." Earth Surface Processes and Landforms 42 (3):503-15. doi: 10.1002/esp.4086

Morgan, J. A., D. J. Brogan, and P. A. Nelson. 2017. "Application of Structure-fromMotion Photogrammetry in Laboratory Flumes." Geomorphology 276:125-43. doi: 10.1016/j.geomorph.2016.10.021.

Mosbrucker, A. R., J. J. Major, K. R. Spicer, and J. Pitlick. 2017. "Camera System Considerations for Geomorphic Applications of SfM Photogrammetry." Earth Surface Processes and Landforms 42 (6):969-86. doi: 10.1002/esp.4066.

Ockelford, A.-M., and H. Haynes. 2013. "The Impact of Stress History on Bed Structure." Earth Surface Processes and Landforms 38 (7):717-27. doi: 10.1002/esp.3348.

Ouédraogo, M. M., A. Degré, C. Debouche, and J. Lisein. 2014. "The Evaluation of Unmanned Aerial System-based Photogrammetry and Terrestrial Laser Scanning to Generate DEMs of Agricultural Watersheds." Geomorphology 214:339-55. doi: 10.1016/j.geomorph.2014.02.016.

Rice, S. P., and M. A. Church. 2010. "Grain-size Sorting within River Bars in Relation to Downstream Fining along a Wandering Channel." Sedimentology 57 (1):232-51. doi: 10.1111/j.1365-3091.2009.01108.x.

Rieke-Zapp, D. H., R. Rosenbauer, and F. Schlunegger. 2009. "A Photogrammetric Surveying Method for Field Applications." The Photogrammetric Record 24 (125):5-22. doi: 10.1111/j.1477-9730.2008.00515.x. 
Rumsby, B. T., J. Brasington, J. A. Langham, S. J. McLelland, R. Middleton, and G. Rollinson. 2008. "Monitoring and Modelling Particle and Reach-scale Morphological Change in Gravel-bed rivers: Applications and Challenges." Geomorphology 93 (1-2):40-54. doi: 10.1016/j.geomorph.2006.12.017.

Smart, G., M. Duncan, and J. Walsh. 2002. "Relatively Rough Flow Resistance Equations." Journal of Hydraulic Engineering 128 (6):568-78. doi: 10.1061/(ASCE)0733-9429(2002)128:6(568).

Smith, M. W., J. L. Carrivick, and D. J. Quincey. 2015. "Structure from Motion Photogrammetry in Physical Geography." Progress in Physical Geography: Earth and Environment 40 (2):247-75. doi: 10.1177/0309133315615805.

Stähly, S., H. Friedrich, and M. Detert. 2017. "Size Ratio of Fluvial Grains' Intermediate Axes Assessed by Image Processing and Square-Hole Sieving." Journal of Hydraulic Engineering 143 (6). doi: 10.1061/(ASCE)HY.1943-7900.0001286.

Stamatopoulos, C., and C. Fraser. 2014. "Automated Target-Free Network Orientation and Camera Calibration." ISPRS Annals of the Photogrammetry, Remote Sensing and Spatial Information Sciences II-5:339-46. doi: 10.5194/isprsannals-II-5-3392014.

Wang, C.-K., J.-T. Chung, and Y.-L. Lin. 2015. "DEM Measurements of a Gravel-Bed Surface Using Two Scales of Images." The Photogrammetric Record 30 (152):387-401. doi: 10.1111/phor.12125.

Westoby, M. J., J. Brasington, N. F. Glasser, M. J. Hambrey, and J. M. Reynolds. 2012. "'Structure-from-Motion' Photogrammetry: A Low-cost, Effective Tool for Geoscience Applications." Geomorphology 179 (0):300-14. doi: 10.1016/j.geomorph.2012.08.021.

Westoby, M. J., S. A. Dunning, J. Woodward, A. S. Hein, S. M. Marrero, K. Winter, and D. E. Sugden. 2015. "Sedimentological Characterization of Antarctic Moraines Using UAVs and Structure-from-Motion Photogrammetry." Journal of Glaciology 61 (230):1088-102. doi: 10.3189/2015JoG15J086.

Woodget, A. S., P. E. Carbonneau, F. Visser, and I. P. Maddock. 2015. "Quantifying Submerged Fluvial Topography Using Hyperspatial Resolution UAS Imagery and Structure from Motion Photogrammetry." Earth Surface Processes and Landforms 40 (1):47-64. doi: 10.1002/esp.3613.

Zhang, Z. 1998. "A Flexible New Technique for Camera Calibration." IEEE Transactions on Pattern Analysis and Machine Intelligence 22(11):1330-4. doi: 10.1109/34.888718. 